\title{
Letter: Response to letter "The exact number of misplacements is essential for the reproducibility of statistical findings by Mansoor et al." by G. Kerry
}

\author{
Nadia Mansoor ${ }^{1}[$ (1)
}

Received: 9 February 2021 / Accepted: 24 May 2021 / Published online: 8 July 2021

(c) The Author(s), under exclusive licence to Springer-Verlag GmbH Austria, part of Springer Nature 2021

\section{Dear colleague,}

We thank you for your interest in our paper. The correct percentage for the Kakarla 1 group in Table 1 is $50.0 \%$ and not $49.4 \%$. Available imaging from CT scans to evaluate Kakarla score was available for 170 out of 211 patients, and 85 patients out of 170 were characterised to have a Kakarla 1 score from postoperative imaging. The exact number of misplacements of EVDs (both tunnelated and bolt) resulting in revision surgery is given in Table 3 , indicating number of misplacements to be $10(4.7 \%)$ in the tunnelated group and $2(3.3 \%)$ in the bolt group. Please note that these numbers correspond to misplacements resulting in revision surgery. A Kakarla score of 3 can still result in a functional drain (as described in the text), and thus, not all suboptimal drains (Kakarla 3) were necessarily revised.

With kind regards,

Nadia Mansoor.

Publisher's Note Springer Nature remains neutral with regard to jurisdictional claims in published maps and institutional affiliations.

This article is part of the Topical Collection on CSF Circulation

Nadia Mansoor

nadia.mauland.mansoor@stolav.no

1 Department of Neurosurgery, St. Olav's University Hospital, Trondheim, Norway 